\title{
Analysis of antagonistic co-contractions with motorized passive movement device in patients with parkinson's disease
}

\author{
Yuri Kwon ${ }^{\text {a }}$, Ji-Won Kim ${ }^{\text {a }}$, Yeji Ho ${ }^{\text {a }}$, Hyeong-Min Jeon ${ }^{\text {a }}$, Min-Jung Bang ${ }^{\text {a }}$, Gwang-Moon \\ Eom $^{\mathrm{a}, \mathrm{b}, *}$ and Seong-Beom Koh ${ }^{\mathrm{c}, *}$ \\ ${ }^{a}$ Biomedical Engineering, Konkuk University, Choongju 380-701, Korea \\ ${ }^{b}$ BK21Plus Research Institute of Biomedical Engineering, Konkuk University, Choongju 380-701, \\ Korea \\ ${ }^{c}$ Department of Neurology, Korea University College of Medicine, Seoul, Korea
}

\begin{abstract}
Patients with Parkinson's disease (PD) suffer from an increased resistance to passive movement of a joint, called as rigidity. Stretch reflex and shortening reaction were suggested to be associated to the rigidity, however, the mechanism is still poorly understood. We hypothesized that the co-contraction of antagonistic muscle pairs is enhanced in patients with PD and this induces resistance persisting throughout its range of motion. To test the hypothesis, we developed a motorized device for application of passive movement of the wrist joint and investigated the co-contraction of muscles during passive movement. It consisted of a servo motor connected to a rotating axis with a timing belt, load cell for the measurement of resistance, and other elements for the fixation of arm and hand. Repetitive passive movement was applied to the wrist joint of patients. Cocontraction of antagonistic muscle pairs was significantly greater in patients than in normal subjects $(\mathrm{p}<0.001)$, suggesting that the enhanced co-contraction is associated with the mechanical resistance during passive movement, i.e. rigidity. Cocontraction during extended state was greater than the other states $(p<0.001)$, which implies that the length-feedback mechanism may play the important role in co-contraction.
\end{abstract}

Keywords: Parkinson's disease, rigidity, co-contraction, motorized device, passive movement

\section{Introduction}

Patients with Parkinson's disease (PD) suffer from an increased resistance to passive movement of a joint, characterized by a continuous and uniform increase in resistance throughout the whole range of motion, called as rigidity [1,2]. Parkinsonian rigidity is widely described as a "lead-pipe" resistance distinguished from spasticity and the other types of abnormal muscle tone.

In the previous study, viscoelastic properties of wrist joint could quantify rigidity for clinical application [3]. Despite decades of investigation, underlying mechanism of rigidity is poorly understood.

\footnotetext{
${ }^{*}$ Corresponding authors: Gwang-Moon Eom, Biomedical Engineering, Konkuk University, Choongju 380-701, Korea. Tel.: +82-43-840-3766; Fax: +82-43-851-0620; E-mail: gmeom@kku.ac.kr.

Seong-Beom Koh, Department of Neurology, Korea University College of Medicine, Seoul, Korea. Tel.: +82-2-2626-3169; E-mail: parkinson@korea.ac.kr.
} 
Long-latency (around 60ms) stretch-reflexes have been demonstrated to be exaggerated in patients with PD [4,5], but the long-latency stretch-reflex alone cannot account for the uniformity of resistance with changing joint angle [6].

Xia et al. suggested that the muscle activation in passively shortened muscle, called as "shortening reaction", also took an important role in the uniform resistance $[7,8]$. They calculated the ratio of electromyogram (EMG) in stretched muscles to the EMG in shortened muscle, and found that the mechanical work done by resistance was partly correlated with the EMG ratio. From this, they suggested that the rigidity caused by the stretch reflex and the stretch reflex is partly compensated by the shortening reaction. However, the correlation was significant only for part of the experimental conditions. Moreover, EMG magnitude was normalized by the resting state EMG of the same muscle so that the torque magnitude corresponding to a certain EMG magnitude would be different between different muscles. Then, the EMG ratio cannot represent the net resistance torque because the normalized EMG of stretched muscle and that of shortened muscle are coming from different muscles.

If the resistance torque is greater than the assistance torque, the resistance torque during the stretching phase should be greater than the assistance torque during the shortening phase in one muscle. In this case, EMG of different phases can be compared directly, because they are coming from the identical muscle and the EMG amplitude is regarded to be correlated to the muscle force or torque even in the dynamic phase as well as in static phase [9]. In our preliminary experiment to confirm this, the EMG ratio of stretch-to-shortening in one muscle was smaller than that for both the flexor and extensor of the wrist. This indicates that the stretch-shortening EMG ratio cannot explain the resistance torque of rigidity.

On the other hand, the co-contraction of antagonistic muscles about a joint was shown to increase the stiffness of the joint and the mechanical resistance, such as the spring and damping constants [10]. This implies that the two antagonistic muscles activated around a joint can be regarded as two mechanical resistances connected to both sides of the segment, and these resistances generate resistance torque irrespective of the movement directions. Therefore, we hypothesized that the co-contraction of antagonistic muscle pairs is enhanced in patients with PD and this induces resistance persisting throughout its range of motion. The purpose of this study is to develop a motorized device for the passive movement of the wrist joint to investigate the co-contraction of muscles during passive movement and to compare the co-contraction of antagonistic muscle pairs in patients and normal subjects.

\section{Materials and methods}

\subsection{Measurement system}

Figure 1 shows the developed motorized device for the application of passive movement. The size of the motorized device was $270 \times 575 \times 155 \mathrm{~mm}^{3}$. A servo motor (APM-SA01ACN, AC servo motor, Korea) was mounted on one side of arm-plate. A timing belt was used to connect the servo motor to the crankshaft with a gear ratio of 2:1. Wrist joint angle was measured by an encoder in the servomotor.

A load cell (CB1-G600, Dacell, Korea) was used to measure the resistant force caused by rigidity of subject. The load cell was positioned between the hand-plate and the under-plate connected to the crankshaft so that the tangential resistance of a hand to the rotational movement of a crankshaft can be measured. Measured tangential force was converted to torque by multiplying the moment of arm of the load cell. The maximum capacity of the load cell was $0.6 \mathrm{~kg}$ and the moment arm (the distance be- 


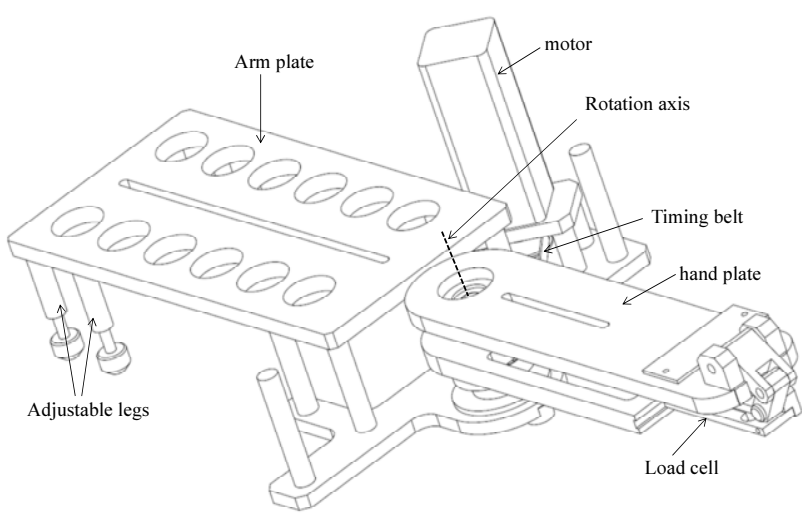

(a) schematic view

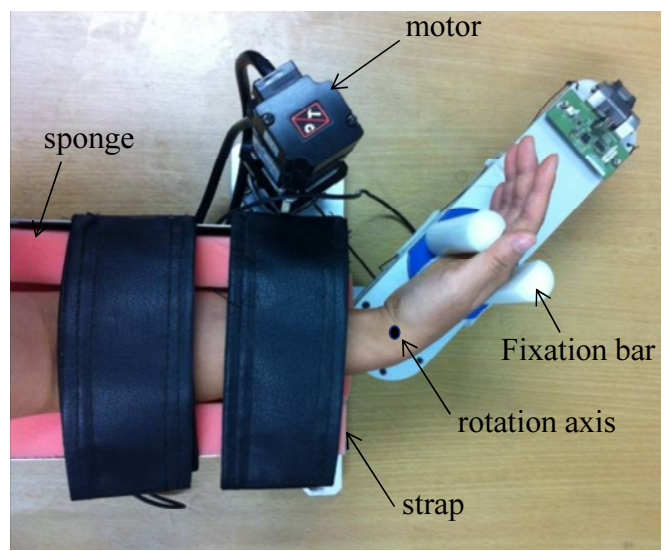

(b) fixation of arm and hand

Fig. 1. Motorized passive movement device.

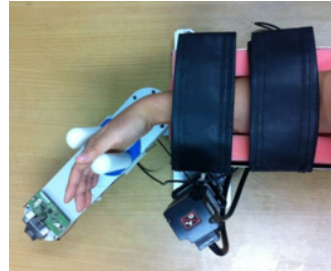

Angle, resistant force

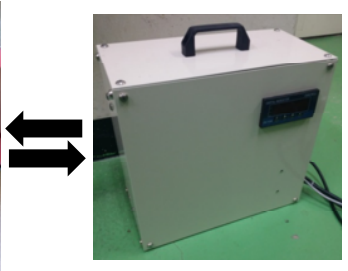

Device controller

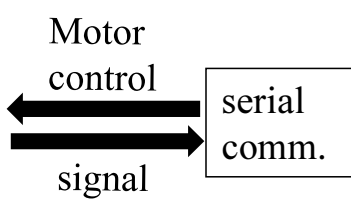

signal

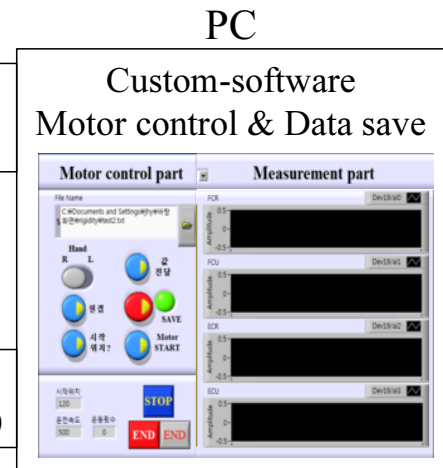

EMG Analog input (DAQ board) sensor receiver (analog)

Wireless EMG system

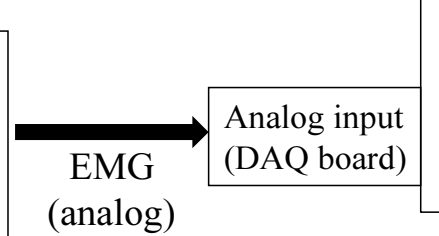

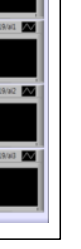

Fig. 2. Structure of whole measurement system.

tween rotation the axes to the load cell) was $0.22 \mathrm{~m}$, resulting in maximum torque of $1.32 \mathrm{Nm}$. Normally the maximum resistant torque was about $0.5 \mathrm{Nm}$, which resides in the maximum measurable torque.

For the fixation of the hand on the hand-plate, two plastic bars were used (Figure 1b). Wrist joint was aligned to the rotation axis. Forearm was mounted in the sponge pad on the arm-plate and was fixed by straps. Legs were adjusted to make the plates be leveled and rubber foot was attached on the bottom of legs for immobilization on the table.

Figure 2 shows the signal flow. Custom-made software coded by LabVIEW (ver. 8.0, NI, TX) in a $\mathrm{PC}$ was used for the signal measurement and control of the device. Wrist angle and the resistant force was measured by the device controller and transmitted to PC through serial communication (RS-232). EMG signals were measured by a wireless EMG system (Trigno wireless system, Delsys, MA). EMG at the receiver was transmitted in analog form to a DAQ system (USB-6215, NI, TX), where the ana$\log$ EMG was converted to digital data and transmitted to PC through USB communication. 


\subsection{Subjects and experiments}

Nineteen patients $(69.1 \pm 9.6 y r s)$ with idiopathic PD and 18 healthy subjects with comparable age $(67.7 \pm 10.9 \mathrm{yrs})$ participated in this study. The clinical rigidity score represents rigidity score of a wrist joint in the unified Parkinson's disease rating scale (UPDRS). The rigidity score of patient was $1.1 \pm 0.8$ which was mild. All subjects provided written informed consents, and ethics committee approval was obtained for this study. Full explanation of the experimental process was given to patients and they were instructed not to exert any voluntary force during the passive movements as in the literature [3].

EMG electrodes were attached to the motor points of four muscles [11]: Flexor Carpi Radialis (FCR), Flexor Carpi Ulnaris (FCU), Extensor Carpi Radialis (ECR) and Extensor Carpi Ulnaris (ECU). ECU is excluded in the further analysis, because it suffered from noise by contact. Any difference in EMG amplitude due to the electrode positioning error was regarded to be compensated with normalization by the maximum EMG amplitude during maximum voluntary contraction (MVC).

The center of wrist joint rotation was set by center of rotation of the crankshaft. Flexion movementpause-extension movement-pause was consecutively imposed at the wrist joint, with eight cycles in one trial, and two trials per subject. Subjects were instructed not to exert any active force during movement.

Figure 3 shows the movement pattern applied to the wrist joint. 6 cycles of extension-flexion movement with rest periods between movements was applied to each patient's wrist. Constant velocity was maintained during movements. When slow movement is imposed, parkinsonian rigidity substantially occurs [12]. Therefore velocity during movement was set to $30 \mathrm{deg} / \mathrm{s}$ for the first half, and $50 \mathrm{deg} / \mathrm{s}$ for the second half of the 8 cycles.

\subsection{Analysis of co-contraction}

EMG was full-wave rectified, and then was passed through Butterworth low pass filter $(\mathrm{Fc}=0.5 \mathrm{~Hz}$, $2^{\text {nd }}$ order). EMG was normalized by the EMG in maximum voluntary contraction (\%MVC). As in Figure 3, EMG signals are classified into four phases by movement direction: 1) extended state, 2) flexion, 3) flexed state, 4) extension.

A co-contraction index (CCI) was used as an index quantifying the extent to which each muscle pair was co-contracting [13]. It includes both the degree to which the co-contracting activation overlaps (overlapping ratio) and the overall activation levels in the muscle pair (overall amplitude) as in Eq. (1).

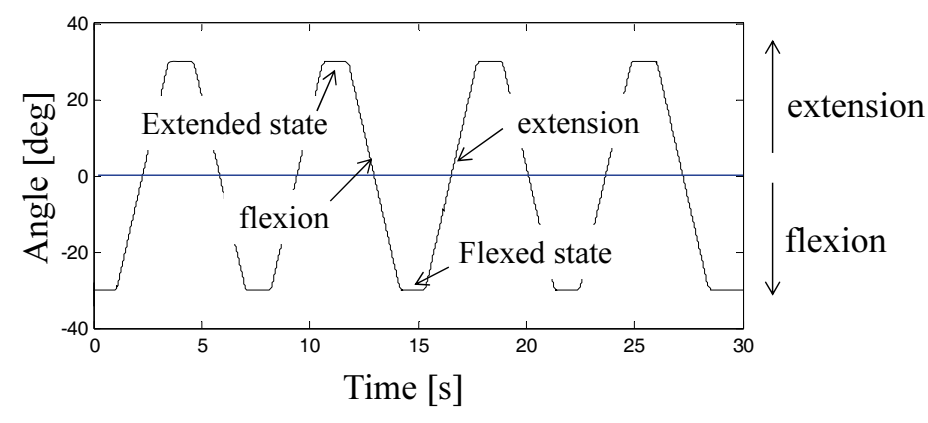

Fig. 3. Applied movement pattern.

(SHR, STR: shortening and stretching phases in case of flexor) 
CCI was calculated for two antagonistic muscle pairs (FCR/ECR pair and FCU/ECR pair) for each phase in each subject group.

$$
C C I=\frac{1}{N} \sum_{i=1}^{N} \frac{E M G_{-} \text {low }(i)}{E M G_{-} \text {high }(i)}\left(E M G_{-} \text {low }(i)+E M G_{-} \text {high }(i)\right)
$$

Differences of CCI for each factor (muscle pairs, group, and phase) were investigated by three-way ANOVA and post hoc test (when an interaction was significant). All statistical analyses were performed using SPSS ver.16 (SPSS Inc., Chicago, IL).

\section{Results and discussion}

Table 1 shows the result of 3-way ANOVA for CCI. Two main effects (group and phase) were significant. The results suggest that the co-contraction of antagonistic muscles acting about the wrist joint is enhanced in patients with PD. The co-contraction of antagonistic muscles about a joint increases the stiffness of the joint and this increases mechanical resistance (such as the spring and damping constant)

Table 1

Three-way ANOVA for CCI (co-contraction index)

\begin{tabular}{lll}
\hline \multirow{3}{*}{ Main effect } & group & $0.000^{* *}$ \\
\cline { 2 - 3 } & phase & $0.000^{* *}$ \\
\cline { 2 - 3 } & muscle pair & 0.155 \\
\hline \multirow{3}{*}{$\begin{array}{l}\text { Interaction } \\
\text { effect }\end{array}$} & group $^{*}$ musc & $0.007^{*}$ \\
\cline { 2 - 3 } & muscle pair $*$ phase $^{*}$ & $0.024^{+}$ \\
\cline { 2 - 3 } & group ${ }^{*}$ phase* muscle pair & 0.971 \\
\hline$* *: \mathrm{p}<0.001, *: \mathrm{p}<0.01,{ }^{+}: \mathrm{p}<0.05$ &
\end{tabular}

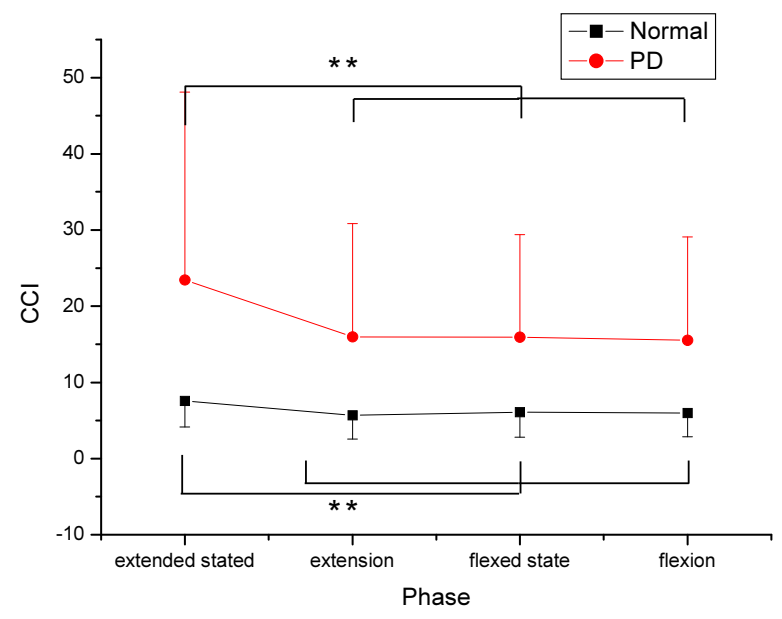

Fig. 4. Post-hoc analysis for group and phase.

${ }^{* *} \mathrm{p}<0.001$. In all phases, CCI was greater in patient group than in normal group $(\mathrm{p}<0.001)$. 
[9]. Therefore, it is highly plausible that the enhanced co-contraction is associated with the passive resistance, rigidity.

There were two interactions of group-phase and group-muscle pair. Detailed structure of the interactions was investigated by post-hoc comparisons shown in Figures 4 and 5.

Figure 4 shows the post-hoc comparisons for group-phase interactions. First, CCI was compared between subject groups in each phase and the result showed that the co-contraction of patients was significantly greater than that of normal subjects in all phases $(\mathrm{p}<0.001)$.

Second, CCI was compared among phases in each subject group, and the result showed that the cocontraction was greater in the extended state than all the other phases in both groups $(p<0.001)$. This suggests that the co-contraction is associated more with the length sensing mechanisms (e.g., Ia in muscle spindle) of flexor muscles that are lengthened during the extended state. It was shown that the resistance during extension is greater than that during flexion of the wrist joint $[3,14]$, which supports that the flexor muscle plays more roles in rigidity.

Figure 5 shows the post-hoc comparisons for group-muscle pair interactions. First, CCI was compared between subject groups in each muscle pair, and the result showed that the co-contraction of patients is greater than that of normal subjects in all muscle pairs $(\mathrm{p}<0.001)$.

Second, CCI was compared between muscle pairs in each subject group. The result showed that the co-contraction was greater in the FCU/ECR pair in the patient group $(p<0.001)$, contrasted by the fact that it was greater in the FCR/ECR pair and normal subject $(p<0.01)$. The difference in these two muscle pairs is the included flexors FCU vs. FCR, which suggests that FCU is more involved in the cocontraction with the extensor in patients. The reason why FCU is more active in the inefficient cocontraction is to be investigated in the further study.

\section{Conclusion}

We developed a motorized device for the passive movement of the wrist joint to investigate the cocontraction of muscles during passive movement. Co-contraction of antagonistic muscle pairs was significantly greater than those of normal subjects especially in the extended state. The results suggest that the enhanced co-contraction of muscles would be associated with the resistance during passive

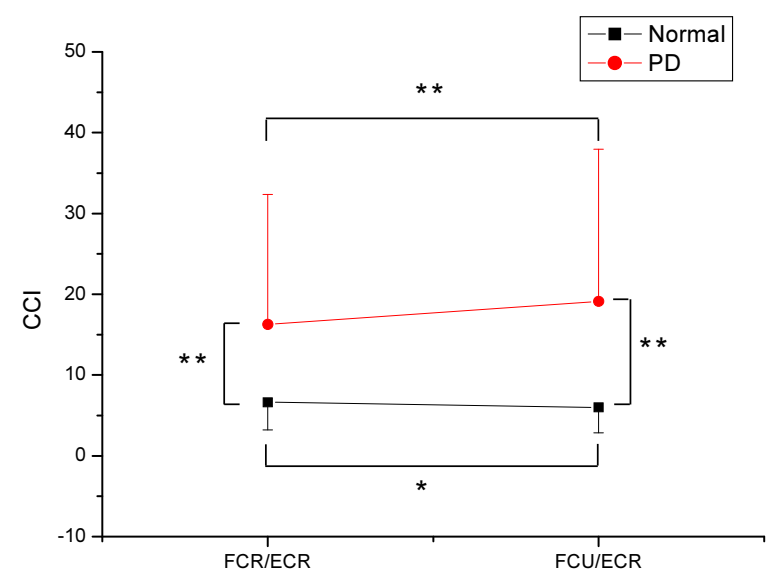

Fig. 5. Post-hoc analysis for group and muscle pair. $\left({ }^{*} \mathrm{p}<0.01,{ }^{* *} \mathrm{p}<0.001\right)$ 
movement.

\section{Acknowledgement}

This paper was supported by the National Research Foundation (NRF) funded by the Ministry of Education, Korea (No. 2011-0015824).

\section{References}

[1] V.S. Fung, J.A. Burne and J.G. Morris, Objective quantification of resting and activated parkinsonian rigidity: A comparison of angular impulse and work scores, Mov. Disord. 15 (2000), 48-55.

[2] M. Hallett, Parkinson revisited: Pathophysiology of motor signs, Advances in Neurology 91 (2003), 19-28.

[3] B.K. Park, Y. Kwon, J.W. Kim, J.H. Lee, G.M. Eom, S.B. Koh, J.H. Jun and J. Hong, Analysis of viscoelastic properties of wrist joint for quantification of parkinsonian rigidity, IEEE Trans. Neural. Syst. Rehabil. Eng. 19 (2011), $167-176$.

[4] R.G. Lee and W.G. Tatton, Motor responses to sudden limb displacements in primates with specific CNS lesions and in human patients with motor system disorders, The Canadian Journal of Neurological Sciences 2 (1975), 285-293.

[5] J.C. Rothwell, J.A. Obeso, M.M. Traub and C.D. Marsden, The behaviour of the long-latency stretch reflex in patients with Parkinson's disease, Journal of Neurology, Neurosurgery, and Psychiatry 46 (1983), 35-44.

[6] R. Xia, D. Powell, W.Z. Rymer, N. Hanson, X. Fang and A.J. Threlkeld, Differentiation between the contributions of shortening reaction and stretch-induced inhibition to rigidity in Parkinson's disease, Exp. Brain Res. 209 (2011), 609618.

[7] R. Xia and W.Z. Rymer, The role of shortening reaction in mediating rigidity in Parkinson's disease, Exp. Brain Res. 156 (2004), 524-528.

[8] R. Xia, J. Sun and A.J. Threlkeld, Analysis of interactive effect of stretch reflex and shortening reaction on rigidity in Parkinson's disease, Clin. Neurophysiol. 120 (2009), 1400-1407.

[9] C.H. Yu, S.H. Shin, H.C. Jeong, D.Y. Go and T.K. Kwon, Activity analysis of trunk and leg muscles during whole body tilt exercise, Biomed. Mater. Eng. 24 (2014), 245-254.

[10] Y. Lee and J.A. Ashton-Miller, The effects of gender, level of co-contraction, and initial angle on elbow extensor muscle stiffness and damping under a step increase in elbow flexion moment, Ann. Biomed. Eng. 39 (2011), 25422549.

[11] A. Perotto, Anatomical guide for the electromyographer, 3rd edi, Charles C. Thomas, USA, 1994, pp. 38-55.

[12] C.D. Marsden, Neurophysiology, In: Parkinson's disease, G. Stem, ed., Johns Hopkins University Press, Maryland, USA, 1990, pp. 57-98.

[13] A. Schinkel-Ivy, B.C. Nairn and J.D. Drake, Investigation of trunk muscle co-contraction and its association with low back pain development during prolonged sitting, J. Electromyogr. Kinesiol. 23 (2013), 778-786.

[14] R. Xia, K. Markopoulou, S.E. Puumala and W.Z. Rymer, A comparison of the effects of imposed extension and flexion movements on Parkinsonian rigidity, Clin. Neurophysiol. 117 (2006), 2302-2307. 